\title{
Two multi-cubic functional equations and some results on the stability in modular spaces
}

\author{
Choonkil Park ${ }^{1}$ and Abasalt Bodaghi ${ }^{2 *}$ (D)
}

"Correspondence:

abasalt.bodaghi@gmail.com ${ }^{2}$ Department of Mathematics, Garmsar Branch, Islamic Azad University, Garmsar, Iran Full list of author information is available at the end of the article

\section{Springer}

\begin{abstract}
In this article, we study $n$-variable mappings which are cubic in each variable. We also show that such mappings can be described by an equation, say, multi-cubic functional equation. Furthermore, we study the stability of such functional equations in the modular space $X_{\rho}$ by applying $\Delta_{2}$-condition and the Fatou property (in some cases) on the modular function $\rho$. Finally, we show that, under some mild conditions, one of these new multi-cubic functional equations can be hyperstable.
\end{abstract}

MSC: 39B52; 39B72; 39B82; 46B03

Keywords: Modular space; (Multi)-cubic functional equation; Hyers-Ulam stability

\section{Introduction}

The main motivation for the investigation of the stability of functional equations was given by Ulam in 1940 in his talk at the University of Wisconsin [47], where he presented a number of unsolved problems. Among these was the famous Ulam stability question concerning the stability of group homomorphisms as follows: Let $G$ be a group and let $H$ be a metric group with the metric $d(\cdot, \cdot)$. Given $\epsilon>0$, does there exist a $\delta>0$ such that if a homomorphism $h: G \longrightarrow H$ satisfies the inequality $d(h(x y), h(x) h(y))<\delta$ for all $x, y \in G$, then there exists a homomorphism $g: G \longrightarrow H$ with $d(h(x), g(x))<\epsilon$ for all $x \in G$ ? In other words, we are looking for situations when the homomorphisms are stable, i.e., if a mapping is almost a homomorphism, then there exists a true homomorphism near it. If we turn our attention to the case of functional equations, we can ask the equation: Under what conditions does there exist a true solution near an approximate mapping differing slightly from a functional equation? If the answer is affirmative, we say that the functional equation is stable. Hyers [16] gave a first affirmative answer to the question of Ulam for Banach spaces as follows: Let $X$ and $Y$ be Banach spaces. Suppose that $f: X \longrightarrow Y$ satisfies

$$
\|f(x+y)-f(x)-f(y)\| \leq \epsilon
$$

(c) The Author(s) 2020. This article is licensed under a Creative Commons Attribution 4.0 International License, which permits use sharing, adaptation, distribution and reproduction in any medium or format, as long as you give appropriate credit to the original author(s) and the source, provide a link to the Creative Commons licence, and indicate if changes were made. The images or other third party material in this article are included in the article's Creative Commons licence, unless indicated otherwise in a credit line to the material. If material is not included in the article's Creative Commons licence and your intended use is not permitted by statutory regulation or exceeds the permitted use, you will need to obtain permission directly from the copyright holder. To view a copy of this licence, visit http://creativecommons.org/licenses/by/4.0/. 
for all $x, y \in X$ and for some $\epsilon \geq 0$. Then, there exists a unique additive mapping $T: X \longrightarrow$ $Y$ such that

$$
\|f(x)-T(x)\| \leq \epsilon
$$

for all $x \in X$, and if $f(t x)$ is continuous in $t$ for each fixed $x$, then $f$ is a linear mapping. Later on, various generalizations and extensions of Hyers' result were established by Aoki [1], Th.M. Rassias [44], Găvruța [15], and J.M. Rassias [43] in different versions for the Cauchy (additive) functional equation $A(x+y)=A(x)+A(y)$ in Banach spaces. Since then, the stability problems of various functional equations on miscellaneous normed spaces have been extensively investigated by a number of authors; for instance, see [12-14, 28, 30, 31], and [33]. Moreover, for some results on positive linear operators, we refer to [32] and [34].

Let $V$ be a commutative group, $W$ a linear space, and $n \geq 2$ an integer. Recall from [10] that a mapping $f: V^{n} \longrightarrow W$ is called multi-additive if it is additive (satisfies the Cauchy functional equation) in each variable. Some facts on such mappings can be found in [23] and many other sources. Besides, $f$ is said to be multi-quadratic if it is quadratic (satisfies the quadratic functional equation $Q(x+y)+Q(x-y)=2 Q(x)+2 Q(y))$ in each variable [11]. In [50], Zhao et al. proved that the mapping $f: V^{n} \longrightarrow W$ is multi-quadratic if and only if the following relation holds:

$$
\sum_{t \in\{-1,1\}^{n}} f\left(x_{1}+t x_{2}\right)=2^{n} \sum_{j_{1}, j_{2}, \ldots, j_{n} \in\{1,2\}} f\left(x_{1 j_{1}}, x_{2 j_{2}}, \ldots, x_{n j_{n}}\right)
$$

where $x_{j}=\left(x_{1 j}, x_{2 j}, \ldots, x_{n j}\right) \in V^{n}$ with $j \in\{1,2\}$. In [10] and [11], Ciepliński studied the generalized Hyers-Ulam stability of multi-additive and multi-quadratic mappings in Banach spaces, respectively (see also [50]). The Jensen-type multi-quadratic mappings and their characterization can be found in [46].

The cubic functional equation

$$
f(x+2 y)-3 f(x+y)+3 f(x)-f(x-y)=6 f(y)
$$

was introduced by J.M. Rassias in [43] for the first time. He investigated the Ulam-Hyers stability problem for this functional equation. After that, the next alternative cubic functional equation

$$
f(2 x+y)+f(2 x-y)=2 f(x+y)+2 f(x-y)+12 f(x)
$$

has been introduced by Jun and Kim in [17]. They also presented the cubic functional equation

$$
f(x+2 y)+f(x-2 y)+6 f(x)=4 f(x+y)+4 f(x-y),
$$

found its general solution, and studied the Hyers-Ulam stability problem for it [18]. For other forms of the (generalized) cubic functional equations and their stability on the various Banach spaces, we refer to [3-7, 29, 35, 36], and [49]. Recently, the stability of multicubic and multi-quartic mappings in Banach spaces via the fixed point method were investigated in [9] and [8], respectively. 
The concept of modular spaces was first introduced by Nakano [39], and then Luxemburg [24] and Mazur, Musielak and Orlicz [27, 37, 38] developed it extensively. Since then, the theory of modulars and modular spaces is widely applied in the study of interpolation theory [22, 26] and various Orlicz spaces [40]. A modular yields less properties than a norm, but it makes more sense in many special situations. When we work in a modular space, it is frequently assumed that the modular satisfies extra additional properties like some relaxed continuity or some $\Delta_{2}$-condition. As for the mentioned condition, Khamsi [19] studied the stability of quasicontraction mappings in modular spaces without $\Delta_{2}$ condition by using the fixed point theorem. Concerning the stability theory in modular spaces, Sadeghi [45] has established generalized Hyers-Ulam stability via the Khamsi fixed point method of a generalized Jensen functional equation $f(r x+s y)=r g(x)+\operatorname{sh}(y)$ in convex modular spaces with the Fatou property satisfying the $\Delta_{2}$-condition with $0<\kappa \leq 2$. In addition, the stability of quadratic functional equations in modular spaces satisfying the Fatou property without using the $\Delta_{2}$-condition was investigated in [48]. Park et al. [41] investigated the stability of additive and Jensen-additive functional equations without using the $\Delta_{2}$-condition by a fixed point method. An alternative generalized HyersUlam stability theorem of a modified quadratic functional equation in a modular spaces using $\Delta_{3}$-condition without the Fatou property on a modular function was presented in [20]. Furthermore, a refined stability result and alternative stability results for additive and quadratic functional equations using the direct method in modular spaces are given in [21]. For the stability of mixed additive-quadratic-cubic mappings in modular spaces which were recently studied, we refer to [25].

In this paper we define multi-cubic mappings and include two characterizations of such mappings. In other words, we prove that every multi-cubic mapping can be shown to satisfy a single functional equation, and vice versa. Moreover, we investigate the generalized Hyers-Ulam stability and hyperstability for multi-cubic mappings in modular spaces by applying the direct method. As a direct consequence of our main results, we show that under some mild conditions these new multi-cubic mappings are hyperstable.

\section{Preliminary notations}

In this section, we recall some basic definitions and remarks concerning modular spaces with modular functionals, which are primitive notions corresponding to norms and metrics, as in $[20,27,37]$ :

Definition 2.1 Let $X$ be a linear space over a field $\mathbb{K}(\mathbb{R}$ or $\mathbb{C})$. A generalized function $\rho: X \longrightarrow[0, \infty]$ is called a modular if it satisfies the following three conditions for elements $\alpha, \beta \in \mathbb{K}, x, y \in X$ :

(i) $\rho(x)=0$ if and only if $x=0$;

(ii) $\rho(\alpha x)=\rho(x)$ for all scalar $\alpha$ with $|\alpha|=1$;

(iii) $\rho(\alpha x+\beta y) \leq \rho(x)+\rho(y)$ for all scalar $\alpha, \beta \geq 0$ with $\alpha+\beta=1$.

If condition (iii) is replaced by $\rho(\alpha x+\beta y) \leq \alpha^{t} \rho(x)+\beta^{t} \rho(y)$ when $\alpha^{t}+\beta^{t}=1$ and $\alpha, \beta \geq 0$ with an $t \in(0,1]$, then $\rho$ is called $t$-convex modular. 1 -convex modular are called convex modular. For a modular $\rho$, there corresponds a linear subspace $X_{\rho}$ of $X$, given by $X_{\rho}:=$ $\{x \in X: \rho(\lambda x) \rightarrow 0$ as $\lambda \rightarrow 0\}$. In this case $X_{\rho}$ is called a modular space.

Let $\rho$ be a convex modular. Then, the modular space $X_{\rho}$ can be equipped with a norm called the Luxemburg norm, defined by $\|x\|_{\rho}=\inf \left\{\lambda>0: \rho\left(\frac{x}{\lambda}\right) \leq 1\right\}$. 
Here, we have some observations:

(1) If $\rho$ is a modular on $X$, then $\rho(t x)$ is an increasing function of $t \geq 0$ for each fixed $x \in X$, that is, $\rho(a x) \leq \rho(b x)$ whenever $0 \leq a<b$;

(2) If $\rho$ is a convex modular on $X$ and $|\alpha| \leq 1$, then $\rho(a x) \leq|\alpha| \rho(x)$ for all $x \in X$. In particular, if $\alpha_{j} \geq 0(j=1,2, \ldots, n)$ with $0<\sum_{j=1}^{n} \alpha_{j} \leq 1$, then $\rho\left(\sum_{j=1}^{n} \alpha_{j} x_{j}\right) \leq \sum_{j=1}^{n} \alpha_{j} \rho\left(x_{j}\right)$ for all $x_{j} \in X$.

Definition 2.2 Let $X_{\rho}$ be a modular space and let $\left\{x_{n}\right\}$ be a sequence in $X_{\rho}$. Then

(i) $\left\{x_{n}\right\}$ is $\rho$-convergent to a point $x_{*} \in X_{\rho}$, and we write $x_{n} \stackrel{\rho}{\rightarrow} x_{*}$, if $\rho\left(x_{n}-x_{*}\right) \rightarrow 0$ as $n \rightarrow \infty$;

(ii) $\left\{x_{n}\right\}$ is a $\rho$-Cauchy sequence if for any $\epsilon>0$ one has $\rho\left(x_{n}-x_{m}\right)<\epsilon$ for all sufficiently large $m, n \in \mathbb{N}$;

(iii) A subset $Y \subseteq X_{\rho}$ is called $\rho$-complete if any $\rho$-Cauchy sequence is $\rho$-convergent to a point in $Y$.

Example $2.3([42])$ Let $\psi:[0, \infty) \longrightarrow \mathbb{R}$ be a function defined by $\psi(0)=0$ and $\psi(t)>0$ for all $t>0$, and $\lim _{t \rightarrow \infty} \psi(t)=\infty$. If, moreover, $\psi$ is convex, continuous and nondecreasing, then $\psi$ is called an Orlicz function. For a measure space $\left(X, \sum, \mu\right)$, suppose that $L^{0}(\mu)$ is the set of all measurable functions on $X$. For each $f \in L^{0}(\mu)$, define $\rho_{\psi}(f)=\int_{X} \psi(|f|) d \mu$. Then, $\rho_{\psi}$ is a modular and the corresponding modular space is called an Orlicz space and denoted by

$$
L_{\psi}=\left\{f \in L^{0}(\mu) \mid \rho_{\psi}(\lambda f) \rightarrow 0 \text { as } \lambda \rightarrow 0\right\} .
$$

One can check that $L_{\psi}$ is $\rho_{\psi}$-complete.

A modular function $\rho$ is said to satisfy the $\Delta_{s}$-condition if there exists $\kappa>0$ such that $\rho(s x) \leq \kappa \rho(x)$ for all $x \in X_{\rho}$. Throughout this paper, we say that the constant $\kappa$ is a $\Delta_{s^{-}}$ constant related to $\Delta_{s}$-condition. Suppose that $\rho$ is convex and satisfies $\Delta_{s}$-condition with $\Delta_{s}$-constant $\kappa$. If $\kappa<s$, then $\rho(x) \leq \kappa \rho\left(\frac{x}{s}\right) \leq \frac{\kappa}{s} \rho(x)$, which implies $\rho=0$. Hence, we must have the $\Delta_{s}$-constant $\kappa \geq s$ if $\rho$ is convex modular. It is said that a modular $\rho$ has the Fatou property if and only if $\rho(x) \leq \liminf _{n \rightarrow \infty} \rho\left(x_{n}\right)$ whenever the sequence $\left\{x_{n}\right\}$ is $\rho$-convergent to $x$ in the modular space $X_{\rho}$.

\section{Characterization of multi-cubic mappings}

Motivated by equation (1.3), we consider the functional equation

$$
8 f\left(\frac{x+2 y}{2}\right)+8 f\left(\frac{x-2 y}{2}\right)=4 f(x+y)+4 f(x-y)-6 f(x) .
$$

It is easy to check that $f(x)=a x^{3}$ is a solution of (3.1). Hence, it said to be a cubic functional equation. The above equation leads us to define $n$-multi-cubic mappings and characterize them in this section.

Throughout this paper, $\mathbb{N}$ stands for the set of all positive integers, $\mathbb{N}_{0}:=\mathbb{N} \cup\{0\}, \mathbb{R}_{+}:=$ $[0, \infty), n \in \mathbb{N}$. For any $l \in \mathbb{N}_{0}, n \in \mathbb{N}, q=\left(q_{1}, \ldots, q_{n}\right) \in\{-2,-1,1,2\}^{n}$ and $x=\left(x_{1}, \ldots, x_{n}\right) \in$ $V^{n}$, we write $l x:=\left(l x_{1}, \ldots, l x_{n}\right)$ and $q x:=\left(q_{1} x_{1}, \ldots, q_{n} x_{n}\right)$, where $l x$ stands, as usual, for the scalar product of an element $l$ on $x$ of the vector space $V$. 
In the sequel, let $V$ and $W$ be vector spaces over the rationals, $n \in \mathbb{N}$ and $x_{i}^{n}=$ $\left(x_{i 1}, x_{i 2}, \ldots, x_{i n}\right) \in V^{n}$, where $i \in\{1,2\}$. We shall denote $x_{i}^{n}$ by $x_{i}$ if there is no risk of ambiguity. Let $x_{1}, x_{2} \in V^{n}$ and $T \in \mathbb{N}_{0}$ with $0 \leq T \leq n$. Put $\mathcal{M}=\left\{\mathfrak{N}_{n}=\left(N_{1}, N_{2}, \ldots, N_{n}\right) \mid N_{j} \in\right.$ $\left.\left\{x_{1 j} \pm x_{2 j}, x_{1 j}\right\}\right\}$, where $j \in\{1, \ldots, n\}$. Consider

$$
\mathcal{M}_{T}^{n}:=\left\{\mathfrak{N}_{n}=\left(N_{1}, N_{2}, \ldots, N_{n}\right) \in \mathcal{M} \mid \operatorname{Card}\left\{N_{j}: N_{j}=x_{1 j}\right\}=T\right\}
$$

We say that a mapping $f: V^{n} \longrightarrow W$ is $n$-multi-cubic or multi-cubic if $f$ is cubic in each variable (see equation (3.1)). From now on, for such mappings, we use the following notations:

$$
\begin{aligned}
& f\left(\mathcal{M}_{T}^{n}\right):= \sum_{\mathfrak{N}_{n} \in \mathcal{M}_{T}^{n}} f\left(\mathfrak{N}_{n}\right), \\
& f\left(\mathcal{M}_{T}^{n}, z\right):=\sum_{\mathfrak{N}_{n} \in \mathcal{M}_{T}^{n}} f\left(\mathfrak{N}_{n}, z\right) \quad(z \in V) .
\end{aligned}
$$

We say that a mapping $f: V^{n} \longrightarrow W$ satisfies the r-power condition in the $j$ th variable if

$$
f\left(z_{1}, \ldots, z_{j-1}, 2 z_{j}, z_{j+1}, \ldots, z_{n}\right)=2^{r} f\left(z_{1}, \ldots, z_{j-1}, z_{j}, z_{j+1}, \ldots, z_{n}\right),
$$

for all $z_{1}, \ldots, z_{n} \in V^{n}$

Remark 3.1 It is easily verified that if $f$ is a multi-cubic mapping, then it satisfies the 3power condition in all variables. But the converse is not true. Here, by means of an example we show that the 3-power condition in all variables for a mapping $f$ does not imply that it is multi-cubic. Let $(\mathcal{A},\|\cdot\|)$ be a Banach algebra. Fix a vector $a_{0}$ in $\mathcal{A}$ (not necessarily unit). Define the mapping $h: \mathcal{A}^{n} \longrightarrow \mathcal{A}$ by $h\left(a_{1}, \ldots, a_{n}\right)=\prod_{j=1}^{n}\left\|a_{j}\right\|^{3} a_{0}$ for $\left(a_{1}, \ldots, a_{n}\right) \in \mathcal{A}^{n}$. It is easily verified that the mapping $h$ satisfies the 3 -power condition in all variables but is not multi-cubic even for $n=1$, that is, $h$ does not satisfy equation (3.1).

In what follows, $\left(\begin{array}{l}n \\ k\end{array}\right)$ is the binomial coefficient defined for all $n, k \in \mathbb{N}_{0}$ with $n \geq k$ by $n ! /(k !(n-k) !)$. In the upcoming result, we show that a multi-cubic mapping $f: V^{n} \longrightarrow W$ has the form

$$
8^{n} \sum_{q \in\{-2,2\}^{n}} f\left(\frac{x_{1}+q x_{2}}{2}\right)=\sum_{k=0}^{n} 4^{n-k}(-6)^{k} f\left(\mathcal{M}_{k}^{n}\right)
$$

for all $x_{1}, x_{2} \in V^{n}$, where $f\left(\mathcal{M}_{k}^{n}\right)$ is defined in (3.2).

Theorem 3.2 Consider the mapping $f: V^{n} \longrightarrow W$. Then, the following assertions are equivalent:

(i) $f$ is multi-cubic;

(ii) $f$ satisfies equation (3.3) with the 3-power condition in all variables.

Proof (i) $\Rightarrow$ (ii) We first note that it is not hard to show that $f$ satisfies the 3-power condition in all variables. Assume that $f$ is multi-cubic. We prove that $f$ satisfies equation (3.3) 
by induction on $n$. For $n=1$, it is trivial that $f$ satisfies equation (3.1). If (3.3) is valid for some positive integer $n>1$, then

$$
\begin{aligned}
8^{n+1} \sum_{q \in\{-2,2\}^{n+1}} f\left(\frac{x_{1}^{n+1}+q x_{2}^{n+1}}{2}\right)= & 48^{n} \sum_{q \in\{-2,2\}^{n}} f\left(\frac{x_{1}^{n}+q x_{2}^{n}}{2}, x_{1 n+1}+x_{2 n+1}\right) \\
& +4 \times 8^{n} \sum_{q \in\{-2,2\}^{n}} f\left(\frac{x_{1}^{n}+q x_{2}^{n}}{2}, x_{1 n+1}-x_{2 n+1}\right) \\
& -6 \times 8^{n} \sum_{q \in\{-2,2\}^{n}} f\left(\frac{x_{1}^{n}+q x_{2}^{n}}{2}, x_{1 n+1}\right) \\
= & 4 \sum_{k=0}^{n} \sum_{q \in\{-2,2\}} 4^{n-k}(-6)^{k} f\left(\mathcal{M}_{k}^{n}, x_{1 n+1}+q x_{2 n+1}\right) \\
& -6 \sum_{k=0}^{n} 4^{n-k}(-6)^{k} f\left(\mathcal{M}_{k}^{n}, x_{1 n+1}\right) \\
= & \sum_{k=0}^{n+1} 4^{n+1-k}(-6)^{k} f\left(\mathcal{M}_{k}^{n+1}\right) .
\end{aligned}
$$

This means that (3.3) holds for $n+1$.

(ii) $\Rightarrow$ (i) Fix $j \in\{1, \ldots, n\}$ and put

$$
\begin{aligned}
f^{*}\left(x_{1 j}, x_{2 j}\right):= & f\left(x_{11}, \ldots, x_{1 j-1}, x_{1 j}+x_{2 j}, x_{1 j+1}, \ldots, x_{1 n}\right) \\
& +f\left(x_{11}, \ldots, x_{1 j-1}, x_{1 j}-x_{2 j}, x_{1 j+1}, \ldots, x_{1 n}\right)
\end{aligned}
$$

Putting $x_{2 k}=0$ for all $k \in\{1, \ldots, n\} \backslash\{j\}$ in (3.3) and using the assumption, we get

$$
\begin{aligned}
\frac{2^{n-1}}{8^{n-1}} & \times 8^{n} f\left(x_{11}, \ldots, x_{1 j-1}, \frac{x_{1 j}+2 x_{2 j}}{2}, x_{1 j+1}, \ldots, x_{1 n}\right) \\
& +\frac{2^{n-1}}{8^{n-1}} \times 8^{n} f\left(x_{11}, \ldots, x_{1 j-1}, \frac{x_{1 j}-2 x_{2 j}}{2}, x_{1 j+1}, \ldots, x_{1 n}\right) \\
= & 2^{n-1} \times 8^{n} f\left(\frac{x_{11}}{2}, \ldots, \frac{x_{1 j-1}}{2}, \frac{x_{1 j}+2 x_{2 j}}{2}, \frac{x_{1 j+1}}{2}, \ldots, \frac{x_{1 n}}{2}\right) \\
& +2^{n-1} \times 8^{n} f\left(\frac{x_{11}}{2}, \ldots, \frac{x_{1 j-1}}{2}, \frac{x_{1 j}-2 x_{2 j}}{2}, \frac{x_{1 j+1}}{2}, \ldots, \frac{x_{1 n}}{2}\right) \\
= & 2^{n-1} \times 4^{n} f^{*}\left(x_{1 j}, x_{2 j}\right) \\
& +\sum_{k=1}^{n-1}\left[\left(\begin{array}{c}
n-1 \\
k-1
\end{array}\right) 4^{n-k} \times 2^{n-k} \times(-6)^{k}\right] f\left(x_{11}, \ldots, x_{1 n}\right) \\
& +\sum_{k=1}^{n-1}\left[\left(\begin{array}{c}
n-1 \\
k
\end{array}\right) 4^{n-k} \times 2^{n-k-1} \times(-6)^{k}\right] f^{*}\left(x_{1 j}, x_{2 j}\right) \\
& +(-6)^{n} f\left(x_{11}, \ldots, x_{1 n}\right)
\end{aligned}
$$




$$
\begin{aligned}
= & {\left[2^{n-1} \times 4^{n}+\sum_{k=1}^{n-1}\left[\left(\begin{array}{c}
n-1 \\
k
\end{array}\right) 4^{n-k} \times 2^{n-k-1} \times(-6)^{k}\right]\right] f^{*}\left(x_{1 j}, x_{2 j}\right) } \\
& +\left[(-6)^{n}+\sum_{k=1}^{n-1}\left[\left(\begin{array}{c}
n-1 \\
k-1
\end{array}\right) 8^{n-k} \times(-6)^{k}\right]\right] f\left(x_{11}, \ldots, x_{1 n}\right) .
\end{aligned}
$$

On the other hand, we have

$$
\begin{aligned}
2^{n-1} & \times 4^{n}+\sum_{k=1}^{n-1}\left(\begin{array}{c}
n-1 \\
k
\end{array}\right) 4^{n-k} \times 2^{n-k-1} \times(-6)^{k} \\
& =2^{n-1} \times 4^{n}+4 \sum_{k=1}^{n-1}\left(\begin{array}{c}
n-1 \\
k
\end{array}\right) 4^{n-k-1} \times(-3)^{k} \\
& =4 \times 2^{n-1}(4-3)^{n-1}=4 \times 2^{n-1} .
\end{aligned}
$$

In addition,

$$
\begin{aligned}
(-6)^{n}+\sum_{k=1}^{n-1}\left(\begin{array}{l}
n-1 \\
k-1
\end{array}\right) 8^{n-k} \times(-6)^{k} & =(-6)^{n}+\sum_{k=0}^{n-2}\left(\begin{array}{c}
n-1 \\
k
\end{array}\right) 8^{n-k-1} \times(-6)^{k+1} \\
& =(-6)^{n}+(-6) \sum_{k=0}^{n-2}\left(\begin{array}{c}
n-1 \\
k
\end{array}\right) 8^{n-k-1}(-6)^{k} \\
& =(-6)^{n}-6\left(2^{n-1}-(-6)^{n-1}\right)=-6 \times 2^{n-1} .
\end{aligned}
$$

Relations (3.5), (3.7), and (3.6) imply that

$$
\begin{aligned}
8 f( & \left.x_{11}, \ldots, x_{1 j-1}, \frac{x_{1 j}+2 x_{2 j}}{2}, x_{1 j+1}, \ldots, x_{1 n}\right) \\
& +8 f\left(x_{11}, \ldots, x_{1 j-1}, \frac{x_{1 j}-2 x_{2 j}}{2}, x_{1 j+1}, \ldots, x_{1 n}\right) \\
= & 4 f^{*}\left(x_{1 j}, x_{2 j}\right)-6 f\left(x_{11}, \ldots, x_{1 n}\right) .
\end{aligned}
$$

This means that $f$ is cubic in the $j$ th variable. Since $j$ is arbitrary, we obtain the desired result.

\section{An alternative characterization of multi-cubic mappings}

It is easy to check that equation (1.2) implies that $f\left(\frac{x}{2}\right)=\frac{1}{8} f(x)$. On the other hand, the equation

$$
8 f\left(\frac{2 x+y}{2}\right)+8 f\left(\frac{2 x-y}{2}\right)=2 f(x+y)+2 f(x-y)+12 f(x)
$$

shows that $f(2 x)=8 f(x)$. Summing up, we have

Proposition 4.1 A mapping $f: V \longrightarrow W$ satisfies equation (1.2) if and only if it satisfies equation (4.1). 
Here, we introduce a different multi-cubic mapping by using equation (4.1). In other words, a mapping $f: V^{n} \longrightarrow W$ is said to be $n$-multi-cubic or multi-cubic if $f$ is cubic in each variable (see equation (4.1)).

Analogous to Theorem 3.2, we show that multi-cubic mappings in the sense above can be unified using an equation without any extra condition on the components as follows:

Theorem 4.2 A mapping $f: V^{n} \longrightarrow W$ is multi-cubic (in the sense above) if and only iff satisfies the equation

$$
8^{n} \sum_{q \in\{-1,1\}^{n}} f\left(\frac{2 x_{1}+q x_{2}}{2}\right)=\sum_{k=0}^{n} 2^{n-k} 12^{k} f\left(\mathcal{M}_{k}^{n}\right)
$$

for all $x_{1}, x_{2} \in V^{n}$, where $f\left(\mathcal{M}_{k}^{n}\right)$ is defined in (3.2).

Proof (Necessity) Assume that $f$ is multi-cubic. We prove that $f$ satisfies equation (4.2) by induction on $n$. For $n=1$, it is trivial that $f$ satisfies equation (4.1). If (4.2) is valid for some positive integer $n>1$, then

$$
\begin{aligned}
8^{n+1} \sum_{q \in\{-1,1\}^{n+1}} f\left(\frac{2 x_{1}^{n+1}+q x_{2}^{n+1}}{2}\right)= & 2 \times 8^{n} \sum_{q \in\{-1,1\}^{n}} f\left(\frac{2 x_{1}^{n}+q x_{2}^{n}}{2}, x_{1 n+1}+x_{2 n+1}\right) \\
& +2 \times 8^{n} \sum_{q \in\{-1,1\}^{n}} f\left(\frac{2 x_{1}^{n}+q x_{2}^{n}}{2}, x_{1 n+1}-x_{2 n+1}\right) \\
& +12 \times 8^{n} \sum_{q \in\{-1,1\}^{n}} f\left(\frac{2 x_{1}^{n}+q x_{2}^{n}}{2}, x_{1 n+1}\right) \\
= & 2 \sum_{k=0}^{n} \sum_{q \in\{-1,1\}} 2^{n-k} 12^{k} f\left(\mathcal{M}_{k}^{n}, x_{1 n+1}+q x_{2 n+1}\right) \\
& +12 \sum_{k=0}^{n} 2^{n-k} 12^{k} f\left(\mathcal{M}_{k}^{n}, x_{1 n+1}\right) \\
= & \sum_{k=0}^{n+1} 2^{n+1-k} 12^{k} f\left(\mathcal{M}_{k}^{n+1}\right) .
\end{aligned}
$$

This means that (4.2) holds for $n+1$.

(Sufficiency) Fix $j \in\{1, \ldots, n\}$ and $f^{*}\left(x_{1 j}, x_{2 j}\right)$ as in (3.4). Putting $x_{2 k}=0$ for all $k \in$ $\{1, \ldots, n\} \backslash\{j\}$ in $(4.2)$, we get

$$
\begin{aligned}
2^{n-1} & \times 8^{n}\left[f\left(x_{11}, \ldots, x_{1 j-1}, \frac{2 x_{1 j}+x_{2 j}}{2}, x_{1 j+1}, \ldots, x_{1 n}\right)\right. \\
& \left.+f\left(x_{11}, \ldots, x_{1 j-1}, \frac{2 x_{1 j}-x_{2 j}}{2}, x_{1 j+1}, \ldots, x_{1 n}\right)\right] \\
= & 2^{n-1} \times 2^{n} f^{*}\left(x_{1 j}, x_{2 j}\right) \\
& +\sum_{k=1}^{n-1}\left[\left(\begin{array}{l}
n-1 \\
k-1
\end{array}\right) 2^{2(n-k)} \times 12^{k}\right] f\left(x_{11}, \ldots, x_{1 n}\right)
\end{aligned}
$$




$$
\begin{aligned}
& +\sum_{k=1}^{n-1}\left[\left(\begin{array}{c}
n-1 \\
k
\end{array}\right) 2^{2(n-k)-1} \times 12^{k}\right] f^{*}\left(x_{1 j}, x_{2 j}\right) \\
& +12^{n} f\left(x_{11}, \ldots, x_{1 n}\right) \\
& =\left[2^{2 n-1} \times 2^{n}+\sum_{k=1}^{n-1}\left[\left(\begin{array}{c}
n-1 \\
k
\end{array}\right) 2^{2(n-k)-1} \times 12^{k}\right]\right] f^{*}\left(x_{1 j}, x_{2 j}\right) \\
& +\left[12^{n}+\sum_{k=1}^{n-1}\left[\left(\begin{array}{c}
n-1 \\
k-1
\end{array}\right) 2^{2(n-k)} \times 12^{k}\right]\right] f\left(x_{11}, \ldots, x_{1 n}\right) .
\end{aligned}
$$

On the other hand, we have

$$
\begin{aligned}
2^{2 n-1}+\sum_{k=1}^{n-1}\left(\begin{array}{c}
n-1 \\
k
\end{array}\right) 2^{2(n-k)-1} \times 12^{k} & =2^{2 n-1}\left(1+\sum_{k=1}^{n-1}\left(\begin{array}{c}
n-1 \\
k
\end{array}\right) 3^{k}\right) \\
& =2^{2 n-1}(1+3)^{n-1}=2^{4 n-3} .
\end{aligned}
$$

Furthermore,

$$
\begin{aligned}
12^{n}+\sum_{k=1}^{n-1}\left(\begin{array}{l}
n-1 \\
k-1
\end{array}\right) 2^{2(n-k)} \times 12^{k} & =12^{n}+\sum_{k=1}^{n-1}\left(\begin{array}{l}
n-1 \\
k-1
\end{array}\right) 2^{2(n-k)} \times 2^{2 k} \times 3^{k} \\
& =12^{n}+3 \times 2^{2 n} \sum_{k=0}^{n-2}\left(\begin{array}{l}
n-1 \\
k-1
\end{array}\right) 3^{k} \\
& =12^{n}+3 \times 2^{2 n}\left(\sum_{k=0}^{n-1}\left[\left(\begin{array}{l}
n-1 \\
k-1
\end{array}\right) 3^{k}\right]-3^{n-1}\right) \\
& =12^{n}+3 \times 2^{2 n}\left((1+3)^{n-1}-3^{n-1}\right) \\
& =12^{n}+3 \times 2^{2 n}\left(2^{2(n-1)}-3^{n-1}\right) \\
& =12 \times 2^{4(n-1)} .
\end{aligned}
$$

Relations (4.3), (4.4), and (4.5) imply that

$$
\begin{aligned}
& 8 f\left(x_{11}, \ldots, x_{1 j-1}, \frac{2 x_{1 j}+x_{2 j}}{2}, x_{1 j+1}, \ldots, x_{1 n}\right) \\
& \quad+8 f\left(x_{11}, \ldots, x_{1 j-1}, \frac{2 x_{1 j}-x_{2 j}}{2}, x_{1 j+1}, \ldots, x_{1 n}\right) \\
& =2 f^{*}\left(x_{1 j}, x_{2 j}\right)+12 f\left(x_{11}, \ldots, x_{1 n}\right) .
\end{aligned}
$$

This means that $f$ is cubic in the $j$ th variable, and so it is a multi-cubic mapping.

\section{Stability results for (3.3)}

In this section, we prove the generalized Hyers-Ulam stability of multi-cubic functional equation (3.3) in the modular space $X_{\rho}$ by applying the $\Delta_{2}$-condition and Fatou property on the modular function $\rho$. 
Here and subsequently, given a mapping $f: V^{n} \longrightarrow W$, we define the difference operator $\Gamma f: V^{n} \times V^{n} \longrightarrow W$ through

$$
\Gamma f\left(x_{1}, x_{2}\right):=8^{n} \sum_{q \in\{-2,2\}^{n}} f\left(\frac{x_{1}+q x_{2}}{2}\right)-\sum_{k=0}^{n} 4^{n-k}(-6)^{k} f\left(\mathcal{M}_{k}^{n}\right)
$$

for $x_{1}, x_{2} \in V^{n}$, where $f\left(\mathcal{M}_{k}^{n}\right)$ is defined in (3.2).

From now on we assume that $V$ is a real vector space and $X_{\rho}$ is a complete modular space satisfying the $\Delta_{2}$-condition, which has the Fatou property unless otherwise stated explicitly. In the next theorem, we establish the stability of functional equation (3.3).

Theorem 5.1 Let $s \in\{1,-1\}$, and let $\phi: V^{n} \times V^{n} \longrightarrow \mathbb{R}_{+}$be a function such that

$$
\sum_{j=\frac{|s-1|}{2}}^{\infty} \frac{\kappa^{3 n|s-1| j}}{2^{3 n j}} \phi\left(2^{s j+1} x_{1}, 2^{s j+1} x_{2}\right)<\infty
$$

for all $x_{1}, x_{2} \in V^{n}$. Suppose that $f: V^{n} \longrightarrow X_{\rho}$ is a mapping satisfying the inequality

$$
\rho\left(\Gamma f\left(x_{1}, x_{2}\right)\right) \leq \phi\left(x_{1}, x_{2}\right)
$$

for all $x_{1}, x_{2} \in V^{n}$. Then, there exists a unique multi-cubic mapping $\mathcal{C}: V^{n} \longrightarrow X_{\rho}$ such that

$$
\rho(f(x)-\mathcal{Q}(x)) \leq \frac{1}{2^{n+\frac{3}{2}|s+1| n}} \sum_{j=\frac{|s-1|}{2}}^{\infty} \frac{\kappa^{3 n|s-1| j} \phi\left(2^{j s+1} x, 0\right)}{2^{3 n j}}
$$

for all $x \in V^{n}$.

Proof We first consider the case $s=1$. Replacing $\left(x_{1}, x_{2}\right)$ by $\left(2 x_{1}, 0\right)$ in (5.2), we have

$$
\rho\left(2^{n} \times 8^{n} f(x)-\sum_{k=0}^{n}\left(\begin{array}{l}
n \\
k
\end{array}\right) 4^{n-k} 2^{n-k}(-6)^{k} f(2 x)\right) \leq \phi(2 x, 0)
$$

for all $x=x_{1} \in V^{n}$. Hence,

$$
\rho\left(16^{n} f(x)-2^{n} f(2 x)\right) \leq \phi(2 x, 0)
$$

for all $x \in V^{n}$. Inequality (5.4) implies that

$$
\rho\left(f(x)-\frac{f(2 x)}{2^{3 n}}\right) \leq \frac{1}{2^{4 n}} \phi(2 x, 0)
$$

for all $x \in V^{n}$. Once more, by induction on $k$, one can prove the following functional inequality:

$$
\rho\left(f(x)-\frac{f\left(2^{k} x\right)}{2^{3 n k}}\right) \leq \frac{1}{2^{4 n}} \sum_{j=0}^{k-1} \frac{\phi\left(2^{j+1} x, 0\right)}{2^{3 n j}}
$$


for all $x \in V^{n}$. Now, interchanging $x$ by $2^{l} x$ in (5.6), we have

$$
\rho\left(\frac{f\left(2^{l} x\right)}{2^{3 n l}}-\frac{f\left(2^{k+l} x\right)}{2^{3 n(k+l)}}\right) \leq \frac{1}{2^{4 n}} \sum_{j=l}^{k+l-1} \frac{\phi\left(2^{j+1} x, 0\right)}{2^{3 n j}}
$$

for all $x \in V^{n}$. Since the right-hand side of the above inequality tends to zero as $l$ goes to infinity, the sequence $\left\{\frac{f\left(2^{k} x\right)}{2^{3 n k}}\right\}$ is a $\rho$-Cauchy sequence in $X_{\rho}$ and so the mentioned sequence is $\rho$-convergent on $X_{\rho}$. Thus, we may define the mapping $\mathcal{C}: V \longrightarrow X_{\rho}$ via $\mathcal{C}(x)=\rho-$ $\lim _{k \rightarrow \infty} \frac{f\left(2^{k} x\right)}{2^{3 n k}}$ for all $x \in V^{n}$. In other words, $\lim _{k \rightarrow \infty} \rho\left(\frac{f\left(2^{k} x\right)}{2^{3 n k}}-\mathcal{C}(x)\right)=0$. Replacing $\left(x_{1}, x_{2}\right)$ by $\left(2^{k} x_{1}, 2^{k} x_{2}\right)$ in (5.2), and dividing the resulting inequality by $2^{3 n k}$, we get

$$
\rho\left(\frac{1}{2^{3 n k}} \Gamma f\left(2^{k} x_{1}, 2^{k} x_{2}\right)\right) \leq \frac{1}{2^{3 n k}} \rho\left(\Gamma f\left(2^{k} x_{1}, 2^{k} x_{2}\right)\right) \leq \frac{1}{2^{3 n k}} \phi\left(2^{k} x_{1}, 2^{k} x_{2}\right)
$$

for all $x_{1}, x_{2} \in V^{n}$. Consider a fixed real number $M$ such that $M \geq 8^{n}+2^{n}$. By the property $\rho(\alpha t) \leq \alpha \rho(t)$ when $0<\alpha \leq 1$, we have

$$
\begin{aligned}
\rho\left(\frac{1}{M} \Gamma \mathcal{C}\left(x_{1}, x_{2}\right)\right)= & \rho\left(\frac{1}{M}\left[\Gamma \mathcal{C}\left(x_{1}, x_{2}\right)-\frac{\Gamma f\left(2^{k} x_{1}, 2^{k} x_{2}\right)}{2^{3 n k}}+\frac{\Gamma f\left(2^{k} x_{1}, 2^{k} x_{2}\right)}{2^{3 n k}}\right]\right) \\
\leq & \frac{8^{n}}{M} \sum_{q \in\{-2,2\}^{n}} \rho\left(\mathcal{C}\left(\frac{x_{1}+q x_{2}}{2}\right)-\frac{1}{2^{3 n k}} f\left(\frac{2^{k}\left(x_{1}+q x_{2}\right)}{2}\right)\right) \\
& +\frac{1}{M} \sum_{k=0}^{n} 4^{n-k}(-6)^{k} \sum_{\mathfrak{N}_{n} \in \mathcal{M}_{T}^{n}} \rho\left(\mathcal{C}\left(\mathfrak{N}_{n}\right)-\frac{f\left(2^{k} \mathfrak{N}_{n}\right)}{2^{3 n k}}\right) \\
& +\frac{1}{M} \rho\left(\frac{\Gamma f\left(2^{k} x_{1}, 2^{k} x_{2}\right)}{2^{3 n k}}\right)
\end{aligned}
$$

for all $x_{1}, x_{2} \in V^{n}$ and all positive integers $k$. Taking the limit as $k \rightarrow \infty$ and using (5.7), we see that $\rho\left(\frac{1}{M} \Gamma \mathcal{C}\left(x_{1}, x_{2}\right)\right)=0$, and hence $\Gamma \mathcal{C}\left(x_{1}, x_{2}\right)=0$ for all $x_{1}, x_{2} \in V^{n}$. This means that $\mathcal{C}$ is a multi-cubic mapping. Now, the Fatou property of modular $\rho$ implies that

$$
\rho(f(x)-\mathcal{C}(x)) \leq \liminf _{\rho \rightarrow \infty}\left(f(x)-\frac{f\left(2^{k} x\right)}{2^{3 n k}}\right) \leq \frac{1}{2^{4 n}} \sum_{j=0}^{k-1} \frac{\phi\left(2^{j+1} x, 0\right)}{2^{3 n j}}
$$

for all $x \in V^{n}$, which shows that the relation (5.3) holds. For the uniqueness of $\mathcal{C}$, we assume that there exists another multi-cubic mapping $\mathcal{C}_{0}: V^{n} \longrightarrow X_{\rho}$ such that

$$
\rho\left(f(x)-\mathcal{C}_{0}(x)\right) \leq \frac{1}{2^{4 n}} \sum_{j=0}^{\infty} \frac{\phi\left(2^{j+1} x, 0\right)}{2^{3 n j}}
$$

for all $x \in V^{n}$ such that $\mathcal{C}_{0}\left(x_{*}\right) \neq \mathcal{C}\left(x_{*}\right)$ for some $x_{*} \in V^{n}$. In other words, there is a positive constant $\delta>0$ such that $\rho\left(\mathcal{C}_{0}\left(x_{*}\right)-\mathcal{C}\left(x_{*}\right)\right)>\delta$. On the other hand, there is a positive integer $p_{0} \in \mathbb{N}$ such that $\frac{1}{2^{4 n}} \sum_{j=p_{0}}^{\infty} \frac{\phi\left(2^{j+1} x, 0\right)}{2^{3 n j}}<\delta$. Since $\mathcal{C}$ and $\mathcal{C}_{0}$ are multi-cubic mappings, we have 
$\mathcal{C}_{0}\left(2^{p_{0}} x\right)=2^{3 n p_{0}} \mathcal{C}_{0}(x)$ and $\mathcal{C}\left(2^{p_{0}} x\right)=2^{3 n p_{0}} \mathcal{C}(x)$. Thus,

$$
\begin{aligned}
\delta<\rho\left(\mathcal{C}_{0}(x)-\mathcal{C}(x)\right) & \leq \frac{1}{2^{3 n p_{0}}} \rho\left(\mathcal{C}_{0}\left(2^{p_{0}} x\right)-f\left(2^{p_{0}} x\right)\right)+\frac{1}{2^{3 n p_{0}}} \rho\left(f\left(2^{p_{0}} x\right)-\mathcal{C}\left(2^{p_{0}} x\right)\right) \\
& \leq \frac{1}{2^{4 n}} \sum_{j=0}^{\infty} \frac{\phi\left(2^{j+1+p_{0}} x, 0\right)}{2^{3 n\left(j+1+p_{0}\right)}}=\frac{1}{2^{4 n}} \sum_{j=p_{0}}^{\infty} \frac{\phi\left(2^{j+1} x, 0\right)}{2^{3 n j}}<\delta,
\end{aligned}
$$

which is a contradiction. Now, assume that $s=-1$. It follows (5.4) that

$$
\rho\left(f(x)-2^{3 n} f\left(\frac{x}{2}\right)\right) \leq \frac{1}{2^{n}} \phi(x, 0)
$$

for all $x \in V^{n}$. By the convexity of the modular $\rho, \Delta_{2}$-condition and (5.9), we have

$$
\begin{aligned}
& \rho\left(f(x)-2^{6 n} f\left(\frac{x}{2^{2}}\right)\right) \\
& \leq \frac{1}{2^{3 n}} \rho\left(2^{3 n} f(x)-2^{6 n} f\left(\frac{x}{2}\right)\right)+\frac{1}{2^{3 n}} \rho\left(2^{6 n} f\left(\frac{x}{2}\right)-2^{9 n} f\left(\frac{x}{2^{2}}\right)\right) \\
& \quad \leq \frac{1}{2^{n}}\left[\frac{\kappa^{3 n}}{2^{3 n}} \phi(x, 0)+\frac{\kappa^{6 n}}{2^{3 n}} \phi\left(\frac{x}{2}, 0\right)\right]
\end{aligned}
$$

for all $x \in V^{n}$. It is routine to show by induction on $k>1$ that

$$
\rho\left(f(x)-2^{3 n k} f\left(\frac{x}{2^{k}}\right)\right) \leq \frac{1}{2^{n}}\left[\sum_{j=1}^{k-1} \frac{\kappa^{3 n(2 j-1)}}{2^{3 n j}} \phi\left(\frac{x}{2^{j-1}}, 0\right)+\frac{\kappa^{6 n(k-1)}}{2^{3 n(k-1)}} \phi\left(\frac{x}{2^{k-1}}, 0\right)\right]
$$

for all $x \in V^{n}$. Replacing $x$ by $\frac{x}{2^{l}}$ in (5.11), we get

$$
\begin{aligned}
& \rho\left(2^{3 n l} f\left(\frac{x}{2^{l}}\right)-2^{3 n(k+l)} f\left(\frac{x}{2^{k+l}}\right)\right) \\
& \leq \kappa^{3 n l} \rho\left(f\left(\frac{x}{2^{l}}\right)-2^{3 n k} f\left(\frac{x}{2^{k+l}}\right)\right) \\
& \leq \frac{\kappa^{3 n l}}{2^{n}}\left[\sum_{j=1}^{k-1} \frac{\kappa^{3 n(2 j-1)}}{2^{3 n j}} \phi\left(\frac{x}{2^{j-1+l}}, 0\right)+\frac{\kappa^{6 n(k-1)}}{2^{3 n(k-1)}} \phi\left(\frac{x}{2^{k-1+l}}, 0\right)\right] \\
& \leq \frac{1}{2^{n}} \frac{2^{3 n l}}{\kappa^{3 n l}}\left[\sum_{j=1+1}^{k+l-1} \frac{\kappa^{3 n(2 j-1)}}{2^{3 n j}} \phi\left(\frac{x}{2^{j-1}}, 0\right)+\frac{\kappa^{3 n(k+l-1)}}{2^{3 n(k+l-1)}} \phi\left(\frac{x}{2^{k-1+l}}, 0\right)\right]
\end{aligned}
$$

for all $x \in V^{n}$. It follows from (5.1) and (5.12) that the sequence $\left\{2^{3 n k} f\left(\frac{x}{2^{k}}\right)\right\}$ is a $\rho$ Cauchy sequence in $X_{\rho}$ and so there exists the mapping $\mathcal{C}: V^{n} \longrightarrow X_{\rho}$ such that $\mathcal{C}(x)=$ $\rho-\lim _{k \rightarrow \infty} 2^{3 n k} f\left(\frac{x}{2^{k}}\right)$. This means that the mentioned sequence is a $\rho$-convergent to $\mathcal{C}(x)$. Using the $\Delta_{2}$-condition without applying the Fatou property, we obtain

$$
\begin{aligned}
\rho(f(x)-\mathcal{C}(x)) & \leq \frac{1}{2^{3 n}} \rho\left(2^{3 n} f(x)-2^{3(k+1) n} f\left(\frac{x}{2^{k}}\right)\right)+\frac{1}{2^{3 n}} \rho\left(2^{3(k+1) n} f\left(\frac{x}{2^{k}}\right)-2^{3 n} \mathcal{C}(x)\right) \\
& \leq \frac{\kappa^{3 n}}{2^{3 n}} \rho\left(f(x)-2^{3 n k} f\left(\frac{x}{2^{k}}\right)\right)+\frac{\kappa^{3 n}}{2^{3 n}} \rho\left(2^{3 n k} f\left(\frac{x}{2^{k}}\right)-\mathcal{C}(x)\right)
\end{aligned}
$$




$$
\begin{aligned}
\leq & \frac{1}{2^{n}} \frac{\kappa^{3 n}}{2^{3 n}}\left[\sum_{j=1}^{k-1} \frac{\kappa^{3 n(2 j-1)}}{2^{3 n j}} \phi\left(\frac{x}{2^{j-1}}, 0\right)+\frac{\kappa^{6 n(k-1)}}{2^{3 n(k-1)}} \phi\left(\frac{x}{2^{k-1}}, 0\right)\right] \\
& +\frac{\kappa^{3 n}}{2^{3 n}} \rho\left(2^{3 n k} f\left(\frac{x}{2^{k}}\right)-\mathcal{C}(x)\right)
\end{aligned}
$$

for all $x \in V^{n}$. Letting $k \rightarrow \infty$, we see that (5.3) holds. The rest of the proof is similar to the previous case. This completes the proof.

The following corollaries are the direct consequences of Theorem 5.1 concerning the stability of (3.3).

Corollary 5.2 Suppose $\theta>0$ and let $r>0$ be such that $r \neq 3 n, \log _{2}^{\frac{\kappa^{6 n}}{2^{3 n}}}$. Let $V$ be a normed space and let $X_{\rho}$ be a $\rho$-complete convex modular space. If $f: V^{n} \longrightarrow X_{\rho}$ is a mapping satisfying

$$
\rho\left(\Gamma f\left(x_{1}, x_{2}\right)\right) \leq \theta \sum_{k=1}^{2} \sum_{j=1}^{n}\left\|x_{k j}\right\|^{r}
$$

for all $x_{1}, x_{2} \in V^{n}$, then there exists a unique multi-cubic mapping $\mathcal{C}: V^{n} \longrightarrow X_{\rho}$ such that

$$
\rho(f(x)-\mathcal{C}(x)) \leq \begin{cases}\frac{2^{r} \theta}{2^{n}\left(2^{3 n}-2^{r}\right)} \sum_{j=1}^{n}\left\|x_{1 j}\right\|^{r} & r \in(0,3 n), \\ \frac{2^{r} \times \kappa^{6 n} \theta}{2^{n}\left(2^{3 n+r}-\kappa^{6 n}\right)} \sum_{j=1}^{n}\left\|x_{1 j}\right\|^{r} & r \in\left(\log _{2}^{\frac{\kappa^{6 n}}{2^{3 n}}}, \infty\right)\end{cases}
$$

for all $x=x_{1} \in V^{n}$.

Proof Putting $\phi\left(x_{1}, x_{2}\right)=\theta \sum_{k=1}^{2} \sum_{j=1}^{n}\left\|x_{k j}\right\|^{r}$ in Theorem 5.1, one can obtain the first and second inequalities for $s=1$ and $s=-1$, respectively.

Here, we have the following observations:

- In the case $s=1$ of Theorem 5.1, we have used the Fatou property, while the $\Delta_{2}$-condition was not applied, and vice versa for the case $s=-1$.

- In Corollary 5.2, if the $\Delta_{2}$-constant is $\kappa=2$, then $\log _{2}^{\frac{\kappa^{6 n}}{2^{3 n}}}=3 n$. Hence, in this case the second condition converts to $r \in(3 n, \infty)$.

Corollary 5.3 Consider a $\delta>0$, let $V$ be a normed space, and let $X_{\rho}$ a $\rho$-complete convex modular space. Iff $: V^{n} \longrightarrow X_{\rho}$ is a mapping satisfying

$$
\rho\left(\Gamma f\left(x_{1}, x_{2}\right)\right) \leq \delta
$$

for all $x_{1}, x_{2} \in V^{n}$, then there exists a unique multi-cubic mapping $\mathcal{C}: V^{n} \longrightarrow X_{\rho}$ such that

$$
\rho(f(x)-\mathcal{C}(x)) \leq \frac{\delta}{2^{n}\left(2^{3 n}-1\right)}
$$

for all $x \in V^{n}$. 
Proof Letting $\phi\left(x_{1}, x_{2}\right)=\delta$ in Theorem 5.1 for the case $s=1$, we establish the desired result.

Recall that a functional equation $\mathcal{F}$ is hyperstable if any mapping $f$ satisfying equation $\mathcal{F}$ approximately is a true solution of $\mathcal{F}$. Under some conditions, the functional equation (3.3) can be hyperstable as follows.

Corollary 5.4 Suppose $\theta>0$, let $V$ be a normed space, and let $X_{\rho}$ be a $\rho$-complete convex modular space. Suppose that $\alpha_{k j}>0$ for $k \in\{1,2\}$ and $j \in\{1, \ldots, n\}$ fulfill $\sum_{k=1}^{2} \sum_{j=1}^{n} \alpha_{k j} \neq$ $3 n, \log _{2}^{\frac{\kappa^{6 n}}{23 n}}$. Iff $: V^{n} \longrightarrow X_{\rho}$ is a mapping satisfying

$$
\rho\left(\Gamma f\left(x_{1}, x_{2}\right)\right) \leq \theta \prod_{k=1}^{2} \prod_{j=1}^{n}\left\|x_{k j}\right\|^{\alpha_{k j}}
$$

for all $x_{1}, x_{2} \in V^{n}$, then it is multi-cubic.

\section{Conclusion}

We studied $n$-variable mappings which are cubic in each variable and showed that such mappings can be characterized by an equation. We also established the stability and hyperstability of such functional equation by using the direct method in the modular spaces.

\section{Conventions}

1 Multi-cubic mapping: An $n$-variable mapping $f$ is multi-cubic if it is cubic in each variable.

2 Stability: A functional equation $\mathcal{F}$ is stable when a mapping that approximately satisfies $\mathcal{F}$ is close to an exact solution of $\mathcal{F}$.

3 Hyperstability: A functional equation $\mathcal{F}$ is hyperstable if any mapping $f$ satisfying equation $\mathcal{F}$ approximately is a true solution of $\mathcal{F}$.

\section{Acknowledgements}

The authors sincerely thank the anonymous reviewers for their careful reading, constructive comments, and suggesting some related references to improve the quality of the first draft.

\section{Funding}

Not applicable.

Availability of data and materials

Not applicable.

\section{Competing interests}

The authors declare that they have no competing interests.

\section{Authors' contributions}

All authors conceived the study, participated in its design and coordination, drafted the manuscript, participated in the sequence alignment, and read and approved the final manuscript.

\section{Author details}

${ }^{1}$ Research Institute for Natural Sciences, Hanyang University, Seoul, Korea. ${ }^{2}$ Department of Mathematics, Garmsar Branch, Islamic Azad University, Garmsar, Iran.

\section{Publisher's Note}

Springer Nature remains neutral with regard to jurisdictional claims in published maps and institutional affiliations. 


\section{References}

1. Aoki, T.: On the stability of the linear transformation in Banach spaces. J. Math. Soc. Jpn. 2, $64-66$ (1950)

2. Bahyrycz, A., Ciepliński, K., Olko, J.: On an equation characterizing multi-additive-quadratic mappings and its Hyers-Ulam stability. Appl. Math. Comput. 265, 448-455 (2015)

3. Bodaghi, A.: Cubic derivations on Banach algebras. Acta Math. Vietnam. 38(4), 517-528 (2013)

4. Bodaghi, A.: Ulam stability of a cubic functional equation in various spaces. Mathematica 55(2), 125-141 (2013)

5. Bodaghi, A.: Intuitionistic fuzzy stability of the generalized forms of cubic and quartic functional equations. J. Intell. Fuzzy Syst. 30, 2309-2317 (2016)

6. Bodaghi, A., Alias, I.A., Ghahramani, M.H.: Approximately cubic functional equations and cubic multipliers. J. Inequal. Appl. 2011, 53 (2011). https://doi.org/10.1186/1029-242X-2011-53

7. Bodaghi, A., Moosavi, S.M., Rahimi, H.: The generalized cubic functional equation and the stability of cubic Jordan *-derivations. Ann. Univ. Ferrara 59, 235-250 (2013)

8. Bodaghi, A., Park, C., Mewomo, O.T.: Multiquartic functional equations. Adv. Differ. Equ. 2019, 312 (2019). https://doi.org/10.1186/s13662-019-2255-5

9. Bodaghi, A., Shojaee, B.: On an equation characterizing multi-cubic mappings and its stability and hyperstability. Fixed Point Theory. To appear. arXiv:1907.09378v2

10. Ciepliński, K.: Generalized stability of multi-additive mappings. Appl. Math. Lett. 23, 1291-1294 (2010)

11. Ciepliński, K.: On the generalized Hyers-Ulam stability of multi-quadratic mappings. Comput. Math. Appl. 62, 3418-3426 (2011)

12. Eshaghi Gordji, M., Bodaghi, A.: On the Hyers-Ulam-Rassias stability problem for quadratic functional equations. East J. Approx. 16(2), 123-130 (2010)

13. Eshaghi Gordji, M., Bodaghi, A., Park, C.: A fixed point approach to the stability of double Jordan centralizers and Jordan multipliers on Banach algebras. UPB Sci. Bull., Ser. A 73(2), 65-74 (2011)

14. Falihi, S., Shojaee, B., Bodaghi, A., Zivari-Kazempour, A.: Approximation on the mixed type additive-quadratic-sextic functional equation. UPB Sci. Bull., Ser. A 81(3), 13-22 (2019)

15. Găvruța, P.: A generalization of the Hyers-Ulam-Rassias stability of approximately additive mappings. J. Math. Anal. Appl. 184, 431-436 (1994)

16. Hyers, D.H.: On the stability of the linear functional equation. Proc. Natl. Acad. Sci. USA 27, $222-224$ (1941)

17. Jun, K.W., Kim, H.M.: The generalized Hyers-Ulam-Russias stability of a cubic functional equation. J. Math. Anal. Appl. 274(2), 267-278 (2002)

18. Jun, K.W., Kim, H.M.: On the Hyers-Ulam-Rassias stability of a general cubic functional equation. Math. Inequal. Appl. 6(2), 289-302 (2003)

19. Khamsi, M.A.: Quasicontraction mappings in modular spaces without $\Delta_{2}$-condition. Fixed Point Theory Appl. 2008 Article ID 916187 (2008)

20. Kim, H.-M., Hong, Y.S.: Approximate quadratic mappings in modular spaces. Int. J. Pure Appl. Math. 116(1), 31-43 (2017)

21. Kim, H.M., Shin, H.Y.: Refined stability of additive and quadratic functional equations in modular spaces. J. Inequal. Appl. 2017, 146 (2017)

22. Krbec, M.: Modular interpolation spaces. Z. Anal. Anwend. 1, 25-40 (1982)

23. Kuczma, M.: An Introduction to the Theory of Functional Equations and Inequalities. Cauchy's Equation and Jensen's Inequality. Birkhäuser, Basel (2009)

24. Luxemburg, W.A.J.: Banach function spaces. PhD Thesis, Delft University of Technology, Delft, The Netherlands (1955)

25. Maghsoudi, M., Bodaghi, A., Niazi Motlagh, A., Karami, M.: Almost additive-quadratic-cubic mappings in modular spaces. Rev. Unión Mat. Argent. 60(2), 359-379 (2019)

26. Maligranda, L.: Orlicz Spaces and Interpolation. Seminarios de Mathematica, vol. 5. Universidade Estadual de Campinas, Departamento de Matematica, Campinas (1989)

27. Mazur, S., Orlicz, W.: On some classes of linear spaces. Stud. Math. 17, 97-119 (1958)

28. Mohiuddine, S.A., Cancan, M., Sevli, H.: Intuitionistic fuzzy stability of a Jensen functional equation via fixed point technique. Math. Comput. Model. 54, 2403-2409 (2011)

29. Mohiuddine, S.A., Rassias, J.M., Alotaibi, A.: Solution of the Ulam stability problem for Euler-Lagrange-Jensen k-cubic mappings. Filomat 30(2), 305-312 (2016)

30. Mohiuddine, S.A., Rassias, J.M., Alotaibi, A.: Solution of the Ulam stability problem for Euler-Lagrange-Jensen k-quintic mappings. Math. Methods Appl. Sci. 40, 3017-3025 (2017)

31. Mohiuddine, S.A., Sevli, H.: Stability of Pexiderized quadratic functional equation in intuitionistic fuzzy normed space. J. Comput. Appl. Math. 235, 2137-2146 (2011)

32. Mursaleen, M., Ansari, K.J.: On the stability of some positive linear operators from approximation theory. Bull. Math. Sci. 5, 147-157 (2015)

33. Mursaleen, M., Ansari, K.J.: The stability of a generalized affine functional equation in fuzzy normed spaces. Publ. Inst. Math. 100(114), 163-181 (2016)

34. Mursaleen, M., Ansari, K.J., Khan, A.: Stability of some positive linear operators on compact disk. Acta Math. Sci. 35(6), $1492-1500$ (2015)

35. Mursaleen, M., Mohiuddine, S.A.: On stability of a cubic functional equation in intuitionistic fuzzy normed spaces Chaos Solitons Fractals 42, 2997-3005 (2009)

36. Mursaleen, M., Mohiuddine, S.A., Ansari, K.J.: On the stability of fuzzy set-valued functional equations. Cogent Math. 4 1281557 (2017)

37. Musielak, J., Orlicz, W.: On modular spaces. Stud. Math. 18, 49-65 (1959)

38. Musielak, J., Orlicz, W.: Some remarks on modular spaces. Bull. Acad. Pol. Sci., Sér. Sci. Math. Astron. Phys. 7, 661-668 (1959)

39. Nakano, H.: Modulared Semi-Ordered Linear Spaces. Maruzen, Tokyo (1950)

40. Orlicz, W.: Collected Papers, Vols. I, II. PWN, Warszawa (1988)

41. Park, C., Bodaghi, A., Kim, S.O.: A fixed point approach to stability of additive mappings in modular spaces without $\Delta_{2}$-conditions. J. Comput. Anal. Appl. 24, 1038-1048 (2018) 
42. Park, C., Rasias, J.M., Bodaghi, A., Kim, S.O.: Approximate homomorphisms from ternary semigroups to modular spaces. Rev. R. Acad. Cienc. Exactas Fís. Nat., Ser. A Mat. 113, 2175-2188 (2019)

43. Rassias, J.M.: Solution of the Ulam stability problem for cubic mappings. Glas. Mat. Ser. III 36(1), 63-72 (2001)

44. Rassias, T.M.: On the stability of the linear mapping in Banach space. Proc. Am. Math. Soc. 72(2), 297-300 (1978)

45. Sadeghi, G.: A fixed point approach to stability of functional equations in modular spaces. Bull. Malays. Math. Sci. Soc. 37(2), 333-344 (2014)

46. Salimi, S., Bodaghi, A.: A fixed point application for the stability and hyperstability of multi-Jensen-quadratic mappings. J. Fixed Point Theory Appl. 22, 9 (2020). https://doi.org/10.1007/s11784-019-0738-3

47. Ulam, S.M.: Problems in Modern Mathematics. Science Editions. Wiley, New York (1964)

48. Wongkum, K., Chaipunya, P., Kumam, P.: On the generalized Ulam-Hyers-Rassias stability of quadratic mappings in modular spaces without $\Delta_{2}$-conditions. J. Funct. Spaces 2015, Article ID 461719 (2015)

49. Yang, S.Y., Bodaghi, A., Mohd Atan, K.A.: Approximate cubic $*$-derivations on Banach *-algebras. Abstr. Appl. Anal. 2012, Article ID 684179 (2012). https://doi.org/10.1155/2012/684179

50. Zhao, X., Yang, X., Pang, C.-T.: Solution and stability of the multiquadratic functional equation. Abstr. Appl. Anal. 2013, Article ID 415053 (2013)

\section{Submit your manuscript to a SpringerOpen ${ }^{\mathcal{D}}$ journal and benefit from:}

- Convenient online submission

- Rigorous peer review

Open access: articles freely available online

High visibility within the field

- Retaining the copyright to your article

Submit your next manuscript at $\boldsymbol{~ s p r i n g e r o p e n . c o m ~}$ 